\title{
Psychosocial environment in childhood and body mass index growth over 32 years
}

\section{Elovainio, Marko}

2017-04

Elovainio , M , Pulkki-Raback , L , Hakulinen , C , Lehtimaki , T , Jokinen , E , Ronnemaa , T , Mikkila , V , Tossavainen , P, Jula , A , Hutri-Kahonen , N, Viikari , J , Keltikangas-Jarvinen , L, Raitakari , O \& Juonala, M 2017 , ' Psychosocial environment in childhood and body mass index growth over 32 years ', Preventive Medicine, vol. 97 , pp. 50-55 . https://doi.org/10.1016/j.ypmed.2016.12.023

http://hdl.handle.net/10138/236997

https://doi.org/10.1016/j.ypmed.2016.12.023

publishedVersion

Downloaded from Helda, University of Helsinki institutional repository.

This is an electronic reprint of the original article.

This reprint may differ from the original in pagination and typographic detail.

Please cite the original version. 


\title{
Psychosocial environment in childhood and body mass index growth over 32 years
}

\author{
Marko Elovainio a,b,*, Laura Pulkki-Råback ${ }^{\mathrm{b}, \mathrm{c}}$, Christian Hakulinen ${ }^{\mathrm{b}}$, Terho Lehtimäki ${ }^{\mathrm{d}}$, Eero Jokinen ${ }^{\mathrm{e}}$, \\ Tapani Rönnemaa e,f ${ }^{\text {,f }}$ Vera Mikkilä h,i, Päivi Tossavainen ${ }^{j}$, Antti Jula a , Nina Hutri-Kähönen ${ }^{k}$, Jorma Viikari ${ }^{\text {f,g,l }}$, \\ Liisa Keltikangas-Järvinen ${ }^{\text {b }}$, Olli Raitakari f,g,k,l, Markus Juonala f,g,k,l \\ a National Institute for Health and Welfare, Finland \\ ${ }^{\mathrm{b}}$ IBS, Institute of Behavioural Sciences, University of Helsinki, Finland \\ ${ }^{\mathrm{c}}$ Helsinki Collegium for Advanced Studies, University of Helsinki, Finland \\ d Department of Clinical Chemistry, Fimlab Laboratories and School of Medicine, University of Tampere, Tampere, Finland \\ e The Department of Pediatrics, University of Helsinki, Finland \\ ${ }^{\mathrm{f}}$ Research Centre of Applied and Preventive Cardiovascular Medicine, University of Turku, Finland \\ ${ }^{g}$ Department of Medicine, University of Turku, Turku, Finland \\ h The Department of Food and Environmental Sciences, University of Helsinki, Finland \\ ${ }^{i}$ Department of Children and Adolescents, Oulu University Hospital, PEDEGO Research Group, and Medical Research Center Oulu, University of Oulu, Oulu, Finland \\ j Department of Pediatrics, University of Tampere and Tampere University Hospital, Tampere, Finland \\ ${ }^{\mathrm{k}}$ Department of Clinical Physiology and Nuclear Medicine, Turku University Hospital, Turku, Finland \\ ${ }^{1}$ Division of Medicine, Turku University Hospital, Turku, Finland
}

\section{A R T I C L E I N F O}

\section{Article history:}

Received 11 August 2016

Received in revised form 9 November 2016

Accepted 18 December 2016

Available online 28 December 2016

\section{Keywords:}

Longitudinal

Growth curve

Weight

Obesity

Childhood

Psychological

\begin{abstract}
A B S T R A C T
The psychosocial environment and especially various psychosocial risks in childhood have been shown to predict later negative health behavior and health problems. In this study, we examined whether various psychosocial factor domains in childhood and adolescence: socioeconomic status, the emotional family environment (parental nurturance, life-satisfaction), parental lifestyle, life-events, the child's self-regulatory behavior and the child's social adaptation were associated with body mass index (BMI) trajectories individually by domain and as a cumulative score across domains. The participants were a nationally representative sample of 2016 men and women from the Young Finns study aged 3-18 years at study entry in 1980. Their BMI was measured at six study phases from 1980 to 2012. Their parents reported all the factors related to their psychosocial environment in 1980 . The participants responded to questions on adulthood socioeconomic status in 2007. The accumulation of psychosocial factors in childhood was the main exposure variable. The findings from repeated measures multilevel modeling showed that parental lifestyle and life-events and the more positive cumulative psychosocial factors score were associated with a slower increase in BMI during follow-up (regression coefficient range from -0.06 to $-0.50)$. In conclusion, the psychosocial environment in childhood and adolescence, particularly parental lifestyle and lack of stressful life-events, are associated with a lower increase of BMI.
\end{abstract}

(C) 2017 Elsevier Inc. All rights reserved.

\section{Introduction}

It has been estimated that globally $>2.1$ billion people are overweight, and childhood obesity is becoming a particularly serious public health problem (Nishtar et al., 2014; Ogden et al., 2002). Because obesity is an important cause of excess mortality and morbidity, especially due to cardiovascular disease (Flegal et al., 2005), a rethinking of the causes, enablers, and barriers to maintain normal weight is needed.

\footnotetext{
Abbreviations: $\mathrm{CI}$, confidence interval; BMI, body mass index.

* Corresponding author at: National Institute for Health and Welfare, P.O. Box 30, 00271 Helsinki, Finland.

E-mail address: marko.elovainio@thl.fi (M. Elovainio).
}

Studies have shown that experiences of adverse childhood environments (such as abuse or traumatic events) are associated with later obesity (Danese \& Tan, 2014; Mason et al., 2015). Recently, however, it has been suggested that positive psychosocial factors may also be critical for promoting lifelong health and health behaviors (Boehm \& Kubzansky, 2012; Chida \& Steptoe, 2008). Studies have demonstrated that multiple factors that are already present in childhood, such as emotionally healthy family relations, a safe and stable environment, and positive individual attributes (e.g., the capacity for self-regulation and optimism) are beneficial for later health and health behavior (Kubzansky et al., 2011; Appleton et al., 2013a; Moffitt et al., 2011). Furthermore, cumulative exposures to such experiences are likely to have a more profound influence on child development than single exposures (Evans et al., 
2013; Slopen et al., 2013a), thus providing a more comprehensive picture of early factors leading to future health.

In this study, we examined whether mixed psychosocial factors in childhood (considering both positive factors and a lack of negative factors), across a wide spectrum of exposures, would predict healthier BMI developmental trajectories from childhood to adulthood and a reduced risk of excess BMI over the 32 years of the study. The psychosocial factors measured at study inception were the socioeconomic environment, the family emotional environment, family health behaviors, family stability, the child's self-regulative behavior and the child's social adaptation; these factors were chosen on the basis of previously-established associations with cardiac health outcomes (Kubzansky et al., 2011; Appleton et al., 2013a; Moffitt et al., 2011), but they had not previously been examined in relation to trajectories of body fat over the life-course.

\section{Participants and methods}

\subsection{Study design and sample}

The Cardiovascular Risk in Young Finns Study is an on-going follow-up study of the risk factors for coronary heart disease among Finnish children, adolescents and young adults (Raitakari et al., 2008). The first cross-sectional study was conducted in 1980, when age cohorts of $3,6,9,12,15$, and 18 year-olds were randomly selected on the basis of social-security numbers, resulting in a total of 3596 participants (from an original sample of 4320). At baseline, the number (percentage) of participants in the various age groups, from the youngest to the oldest, was: 557 (16\%), 583 (16.2\%), 646 (18.0\%), $651(18.1 \%), 602(16.7 \%)$ and $537(14.9 \%)$ respectively. The measurement of BMI was performed in 1980 and repeated in follow-ups in $1983,1986,2001,2007$, and 2012. In the final study phase, the participants were 35 to 50 years of age. Age and sex were reported by the participants or their parents (the three youngest cohorts) in 1980. 2874 participants ( 1289 men and 1585 women) provided information on BMI from at least one follow-up assessment, and 2061 participants (1062 women) provided complete data on psychosocial factors. The reasons for the data loss are listed in Table 1. The participants in the present study sample were more likely to be women ( $\mathrm{p}<0.001)$, have a lower BMI $(\mathrm{p}=0.047)$, and higher education in adulthood $(p<0.001)$ than those who dropped out of the study at some earlier point, but no other differences were detected. The characteristics of the study sample are shown in Table 2.

Written informed consent was obtained from participants who were at least 9 years old and from the parents of younger participants. The research plan and data collection procedures were accepted by the ethical review boards of five universities (Helsinki, Turku, Tampere, Kuopio and Oulu), and data collection was conducted according to WHO standards as well as the Helsinki Declaration.

Table 1

Reasons for missing data in the Cardiovascular Risk.

\begin{tabular}{ll}
\hline Reason & $\mathrm{n}$ \\
\hline Total sample in 1980 & 3596 \\
Missing all BMI measures during follow-up or missing any of the childhood $^{\text {psychosocial factors }}{ }^{\mathrm{a}}$ & 1188 \\
Withdrawal from the study $^{\mathrm{b}}$ & 122 \\
Moved abroad & 72 \\
Could not be contacted & 49 \\
Death & 104 \\
Remaining analytic sample & 2061 \\
\hline
\end{tabular}

a Did not return the questionnaire or did not attend to clinical examination.

b Written notification of permanent discontinuation.
Table 2

Childhood and adulthood characteristics. The Cardiovascular Risk in Young Finns Study $(\mathrm{n}=2061)$.

\begin{tabular}{lll}
\hline & Mean $/ \%$ & $\mathrm{SD} / \mathrm{n}$ \\
\hline Age in 1980 & 9.8 & 4.9 \\
Male sex $(\% / \mathrm{n})$ & 49 & 999 \\
Outcomes & & \\
Body-mass index $\left(\mathrm{kg} / \mathrm{m}^{2}\right)$ in 1980 & 17.6 & 3.0 \\
Body-mass index $\left(\mathrm{kg} / \mathrm{m}^{2}\right)$ in 1983 & 18.6 & 3.3 \\
Body-mass index $\left(\mathrm{kg} / \mathrm{m}^{2}\right)$ in 1986 & 20.0 & 3.3 \\
Body-mass index $\left(\mathrm{kg} / \mathrm{m}^{2}\right)$ in 2001 & 24.8 & 4.0 \\
Body-mass index $\left(\mathrm{kg} / \mathrm{m}^{2}\right)$ in 2007 & 25.8 & 4.5 \\
Body-mass index $\left(\mathrm{kg} / \mathrm{m}^{2}\right)$ in 2011 & 26.4 & 5.0 \\
Psychosocial factors in childhood & & \\
Favorable socioeconomic status (scale 0-4) & 1.7 & 1.2 \\
Favorable emotional environment (scale 0-4) & 2.5 & 1.0 \\
Optimal health behaviors of parents (scale 0-6) & 4.9 & 1.2 \\
Lack of stressful events (scale 0-5) & 4.8 & 0.5 \\
Self-regulatory behavior (scale 0-7) & 6.6 & 0.8 \\
Social adjustment (scale 0-2) & 1.5 & 0.7 \\
Favorable psychosocial factor score ${ }^{\mathrm{a}}$ & 0.5 & 2.7 \\
Adulthood socioeconomic position & & \\
Low & 33 & 424 \\
Middle & 47 & 614 \\
High & 20 & 265 \\
\hline
\end{tabular}

a Sum score of the psychosocial factors after converting each psychosocial factor into a z-score.

\subsection{Psychosocial factors in childhood}

As in previous studies (Pulkki-Råback et al., 2015), we examined six psychosocial factors: the socioeconomic environment, the emotional environment, parental health behaviors, stressful events, and the child's self-regulation and social adjustment. These factors were reported by the parents of the study participants using a paper-and-pencil questionnaire at the baseline examination in 1980. Cumulative scores have become common in research on childhood psychosocial factors (Evans et al., 2013). Such models define binary risk factors (risk vs. no risk), which are then summed together. We built the six psychosocial factor domains from binary variables where 1 stands for favorable and 0 for a less than favorable level, as described below, and formed a total (cumulative) score by summing these six psychosocial factors. The six psychosocial factors were:

(1) The socioeconomic factors score, which consisted of four factors: upper white collar occupation (1 point), academic/college degree (1 point), family income in the highest $25 \%$ ( 1 point), and occupational stability as indicated by the absence of unemployment spells/retirement due to disability/long-term sick-leave (1 point). Summing the points together yielded a score from 0 (a less than favorable level in all components) to 4 (a favorable level in all components).

(2) The emotional family environment score, which consisted of four factors. The first was the absence of a self-reported, previously diagnosed, parental mental disorder (1 point). The second was high parental nurturance, measured using a 7-item scale $(\alpha=0.70)$ (Katainen et al., 1997). A reply of "very often" to all the items on the scale (Supplement Table S1) gave 1 point. The third component was high parental life satisfaction $(\alpha=0.70)$, measured using a 3-item scale (Supplement Table S1). A positive reply to all three items gave 1 point. Fourth, reasonable alcohol use was included because of evidence indicating that unhealthy drinking is harmful to emotional development (Johnson et al., 1999). Parents reporting heavy drinking "never or at maximum three times per year" were classified as reasonable users ( 1 point). The range of the total emotional family environment scale was 0-4. 
(3) The health behaviors of the parents, which were assessed separately for the mother and the father. Body-mass index $<30.0 \mathrm{~kg} /-$ me was used as a proxy of excess energy intake $(0=$ overweight, $1=$ not overweight). Other health behaviors were non-smoker (1 point); regular physical activity (1 point for exercise at least once a week). Summing together parental health behaviors resulted in a scale range of $0-6$.

(4) Lack of stressful events, which included events that have previously been associated with cardiac outcomes (Korkeila et al., 2010). They were moving residence, change of school, parental divorce or separation, death of a family member, and serious disease in the family. The lack of each event produced one point. The scale range was $0-5$ points, a higher score indicating less stressful events.

(5) The self-regulatory behavior of the participant, which consisted of two scales, one measuring high self-control and the other measuring high aggression control. The predictive validity of both scales has been previously established (Pulkki-Råback et al., 2005; Keltikangas-Järvinen et al., 2006). The self-control scale consisted of one question (Supplemental Table 1) where children described by their parents as being very controlled "always or most of the time" received 1 point. Aggression control ( $\alpha=0.60$ ) was measured with six items (Supplemental Table 1) each giving 1 point. The total score was formed by combining scores from self-control and aggression control (range 0-7).

(6) Social adjustment, which was reported by the primary caregiver responding to two items: "I am not particularly worried about my child's behavior" ( 1 point) and "My child is well-adjusted and copes well with life's challenges" ( 1 point). Thus, the scale range was from 0 to 2 . Our previous work has shown that these questions predict outcomes theoretically related to social adjustment (Katainen et al., 1997; Pulkki-Raback et al., 2005).

\subsection{Psychosocial factors score (cumulative score)}

Following a previous study using the same cohort (Pulkki-Råback et al., 2015), the six psychosocial factors were converted into standard scores (z-scores) and then summed together to form the overall psychosocial factors score. The formula was: socioeconomic environment + emotional environment + parental health behaviors + lack of stressful events + high self-regulation + social adjustment.

Socioeconomic status in adulthood was examined because it potentially explains the link between childhood factors and adult BMI. Socioeconomic status in adulthood was determined by the education level reported by the participants in 2007 and classified as $1=$ low (comprehensive school); 2 = intermediate (upper-secondary education): $3=$ high (academic, graduated from or studying at a polytechnic or university).

\subsection{Measurements of body mass index}

The participants' body mass index was calculated as weight ( $\mathrm{kg}) /$ height $\left(\mathrm{m}^{2}\right)$. The participants were weighed in light clothing without shoes on a set of digital scales with an accuracy of $0.1 \mathrm{~kg}$, whereas height was measured with a wall-mounted stadiometer accurate to $0.1 \mathrm{~cm}$, as described in detail previously (Raitakari et al., 2008). BMI was available for all age groups at all measurement points. Obesity (BMI $>30 \mathrm{~kg} / \mathrm{m}^{2}$ ) at the last three measurement points, when all the participants were adults, was used in the sensitivity analyses.

\subsection{Statistical analyses}

Linear regression analyses were used to examine the associations between the psychosocial factors score (as a continuous variable) and BMI. To further assess possible discontinuities or threshold effects, the psychosocial factors score was divided into tertiles based on the distribution of the sample.

Random-intercept and random-slope multilevel modeling (i.e., growth curve modeling) were used to examine the growth trajectories of BMI over time as a function of psychosocial factors in childhood. Age and its second-degree polynomial were used as randomly varying time variables, and the continuous psychosocial factors score was entered as the second-level variable predicting change (slope) of BMI across the six measurements. The analyses were conducted hierarchically so that in the first step all the psychosocial factor domains were tested separately (adjusted for age and sex), then simultaneously (adjusted for age and sex) and then simultaneously but additionally adjusted for education in adulthood. We then tested the association between the cumulative psychosocial factors score and the change in BMI adjusted for age and sex and then additionally adjusted for education in adulthood. There were no statistically significant interactions between sex and psychosocial factors in predicting BMI at any of the study phases ( $\mathrm{p}$-values = $0.056,0.074,0.285,0.626,0.576,0.472$ ), and thus the results are reported for men and women combined.

As additional analyses, we used imputation by chained equations to estimate the effect of non-randomly missing data on the associations. We imputed the missing childhood psychosocial factors and the covariates (but not the outcomes) and calculated the pooled estimates over 50 imputed datasets.

As sensitivity analyses, we tested whether the childhood psychosocial factors score was associated with obesity $\left(\mathrm{BMI}>30 \mathrm{~kg} / \mathrm{m}^{2}\right)$ at the last three measurement points, when all the participants were adults.

\section{Results}

Each standard deviation increase in the psychosocial factors score was associated with a lower BMI in 1980, 1983, 1986, 2001, 2007 and 2011 (standardized Betas $=-0.04,-0.05,-0.08,-0.15,-0.18$ and -0.17 , respectively; p-values $=0.030,0.019,0.001,<0.001,<0.001$ and $<0.001$, respectively). Each tertile increase in the psychosocial factors score was associated with a lower BMI, thus suggesting an inverse dose-response association.

The growth-curve analyses showed that BMI increased by age in a curvilinear fashion (age $\mathrm{ag}^{2}$ slope $\mathrm{b}=-0.01, \mathrm{p}<0.001$ ). The full model with all the predictors (age, sex, adulthood socioeconomic position, the psychosocial factors score, and the interaction term between age and the psychosocial factors score) showed that the rise in BMI over time was predicted by psychosocial factors in childhood (psychosocial factors $*$ time-dependent slope of BMI $b=-0.01, p<0.001$ ). This finding is described in Fig. 1, which shows the rate of rise in BMI according to psychosocial factor tertiles. Each tertile increase in favorable psychosocial factors was associated with a slower rise in BMI over time.

Table 3 shows that each increase of one standard deviation in the psychosocial factors score was associated with lower BMI when adjusting for age and sex (Table $3 ; \mathrm{b}=-0.06, \mathrm{p}=0.005, \mathrm{R}^{2}=0.06$ ). When adulthood socioeconomic status was added, the association was still statistically significant $\left(b=-0.06, p<0.012\right.$ ) and the pseudo $R^{2}$ for the model was 0.31 .

When examining the contributions of the six specific psychosocial factor domains, healthy parental lifestyles $(b=-0.21, \mathrm{p}<0.001)$ and lack of stressful events ( $b=-0.37, p<0.001$ ) were associated with lower BMI in separate models. When all the psychosocial factors were examined simultaneously, healthy parental lifestyles $(b=-0.20$, $\mathrm{p}<0.001)$ and lack of stressful events $(b=-0.38, p<0.001)$ remained independent predictors of BMI. These associations were also robust to 


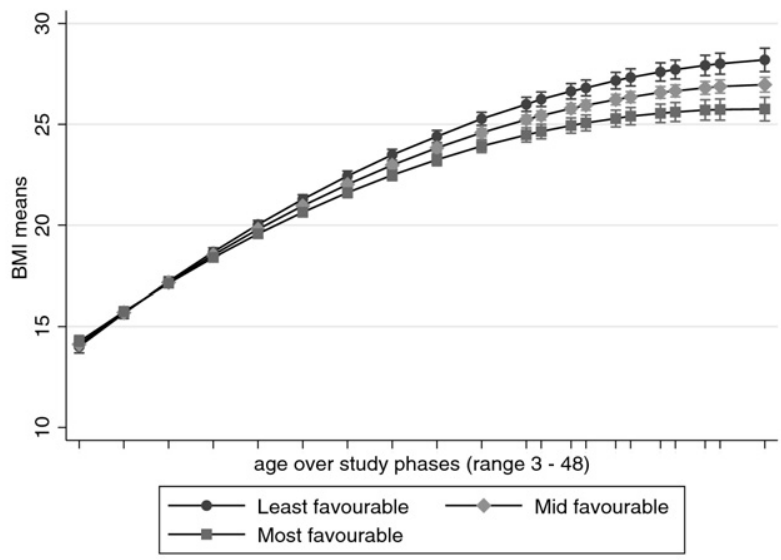

Fig. 1. BMI growth curves for high, median and low accumulations of favorable psychosocial factors in childhood $(n=1164)$, adjusted for age and sex.

adjustments for socioeconomic status in adulthood $(\mathrm{b}=-0.22, \mathrm{p}=$ $0.001 ; \mathrm{b}=-0.50, \mathrm{p}<0.001$ ) (Table 3 ).

The findings in the imputed data were similar in direction and magnitude to the findings in the observed data. Each standard deviation increase in the favorable psychosocial factors score was associated with a lower BMI in 1980, 1983, 1986, 2001, 2007, and 2011 (standardized Betas $=-0.03,-0.03,-0.03,-0.11,-0.13$ and -0.12 ; $\mathrm{p}$-values $=$ $0.065,0.103,0.161,<0.001,<0.001$ and $<0.001)$. Each increase of one standard deviation in the favorable psychosocial factors score was associated with a lower BMI when adjusting for age and sex $(b=-0.05$, $\mathrm{p}=0.001)$. The association was robust to adjustments for all the potential confounders or mediators $(b=-0.03, p=0.028$ ). We also repeated the analyses separately for the three youngest and three oldest age cohorts and a similar, although non-significant, pattern in the associations was found $(b=-0.03$ for younger and $b=-0.04$ for older NS.). We also repeated the analyses using a sex-adjusted and age-specific standardized favorable psychosocial factors score and obtained similar results $(b=-0.04, p=0.031$ ) to as with the group level z-score.

We additionally tested whether the childhood psychosocial factors score was associated with obesity $\left(\mathrm{BMI}>30 \mathrm{~kg} / \mathrm{m}^{2}\right)$ at the last three measurement points, when all the participants were adults. A higher favorable psychosocial factor score predicted a smaller risk of being obese in 2001 (OR 0.87, 95\% CI 0.82-0.92) in 2007 (OR 0.89, 95\% CI 0.84-0.94) and in 2011 (OR 0.89, 95\% CI 0.85-0.94). All the models were adjusted for age and gender. The associations were all robust to adjustment for socioeconomic status in adulthood (ORs 0.87, 0.91, 0.90, respectively).

\section{Discussion}

Our results showed that health behaviors of parents, and lack of stressful life-events in childhood are associated with more optimal body mass development during a time-period from childhood to adulthood. Also the score that reflects the accumulation of the psychosocial factors in childhood was associated with body mass growth, although individual associations between factors, such emotional environment, self-regulatory behavior, and social adjustment showed no significant association. We use the term accumulation as a combination of protective factors at a single time point rather than buildup over time. Children from positive environments had an adult BMI (mean of the three latest study phases) within normal weight $\left(24.9 \mathrm{~kg} / \mathrm{m}^{2}\right)$, while as a group those with the least positive childhoods were clearly overweight as adults, with a mean BMI over the last three study phases of $26.3 \mathrm{~kg} / \mathrm{m}^{2}$. Positive childhood factors were also associated with $10 \%$ less obesity in adulthood. Similar results showing associations correlations between a positive childhood psychosocial environment on and positive cardiovascular and mental health outcomes have been published (Pulkki-Råback et al., 2015; Appleton et al., 2013b) but not previously in relation to the longitudinal development of body weight. The current study expands previous knowledge, as there has been a paucity of longitudinal studies examining the contribution of childhood social determinants to long-term development of overweight and obesity (Slopen et al., 2013b). Pediatricians and other health care professionals working with children and youth have been urged adopt a deeper understanding of the early life origins of health (Garner et al., 2012), and the current findings expand this knowledge by suggesting that promoting positive early-life environments may be a useful step in obesity prevention.

The difference in BMI between the least and the most favorable groups was approximately 2.5 units, which is clinically relevant given that a difference 0.5 units is associated with an approximately $40 \%$ increased risk of type 2 diabetes, a 20\% higher risk of heart failure and $10 \%$ elevation in the risk of coronary artery disease in middle-aged and elderly populations (Vazquez et al., 2007; Hägg et al., 2015). Weight gain during adulthood is a multifactorial outcome, and it is a significant finding if childhood psychosocial factors are associated with significant changes in BMI over such a long time. Of the various childhood indicators examined in this study, the absence of stressful events, which indicates a psychologically safe and stable childhood environment, had the strongest association with optimal body weight. Pediatricians and other health care specialists could make interventions focused on multiple factors, as suggested by the Center for Disease Prevention and Control (CDC) recommendations (www.cdc.gov). These recommendations include not only efforts to enhance specific health behaviors but also the

Table 3

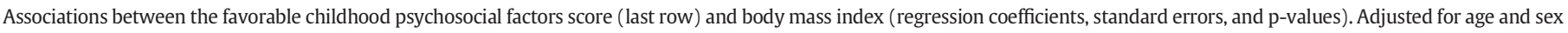
(Models 1 and 2) and additionally for socioeconomic position in adulthood (Models 3 and 4). The Cardiovascular Risk in Young Finns Study ( $\mathrm{n}=2061-1206$ ).

\begin{tabular}{|c|c|c|c|c|c|c|c|c|c|c|c|c|c|c|c|}
\hline \multirow[t]{2}{*}{ Predictors in the model } & \multicolumn{3}{|c|}{ Model 1} & \multicolumn{3}{|c|}{ Model 2} & \multicolumn{3}{|c|}{ Model 3} & \multicolumn{3}{|c|}{ Model 4} & \multicolumn{3}{|c|}{ Model 5} \\
\hline & Beta & (SE) & p-Value & Beta & (SE) & p-Value & Beta & (SE) & p-Value & Beta & (SE) & p-Value & Beta & (SE) & p-Value \\
\hline Age & 0.31 & $(0.01)$ & $<0.001$ & 0.31 & $(0.01)$ & $<0.001$ & 0.29 & $(0.01)$ & $<0.001$ & 0.31 & $(0.01)$ & $<0.001$ & 0.28 & $(0.01)$ & $<0.001$ \\
\hline Male sex & -0.06 & $(0.11)$ & 0.582 & -0.04 & $(0.10)$ & 0.705 & 0.12 & $(0.12)$ & 0.364 & 0.09 & $(0.11)$ & 0.418 & 0.07 & $(0.15)$ & 0.604 \\
\hline Adulthood socioeconomic position & & & & & & & -0.19 & $(0.09)$ & 0.054 & & & & -0.17 & $(0.09)$ & 0.060 \\
\hline \multicolumn{16}{|l|}{$\begin{array}{l}\text { Favorable childhood psychosocial } \\
\text { factors }\end{array}$} \\
\hline a. Socioeconomic status & -0.07 & $(0.05)$ & 0.188 & -0.05 & $(0.06)$ & 0.324 & -0.03 & $(0.06)$ & 0.702 & & & & & & \\
\hline b. Emotional environment & 0.00 & $(0.05)$ & 0.964 & 0.03 & $(0.05)$ & 0.552 & 0.07 & $(0.06)$ & 0.248 & & & & & & \\
\hline c. Health behaviors of parents & -0.21 & $(0.06)$ & $<0.001$ & -0.20 & $(0.06)$ & $<\mathbf{0 . 0 0 1}$ & -0.22 & $(0.07)$ & 0.001 & & & & & & \\
\hline d. Lack of stressful events & -0.37 & $(\mathbf{0 . 0 8 )}$ & $<\mathbf{0 . 0 0 1}$ & -0.38 & $(\mathbf{0 . 1 0})$ & $<\mathbf{0 . 0 0 1}$ & -0.50 & $(\mathbf{0 . 1 0})$ & $<\mathbf{0 . 0 0 1}$ & & & & & & \\
\hline e. Self-regulatory behavior & -0.06 & $(0.05)$ & 0.272 & -0.07 & $(0.06)$ & 0.219 & -0.11 & $(0.08)$ & 0.166 & & & & & & \\
\hline f. Social adjustment & 0.07 & $(0.06)$ & 0.215 & 0.09 & $(0.06)$ & 0.111 & 0.08 & $(0.07)$ & 0.221 & & & & & & \\
\hline Combined favorable psychosocial factor & & & & & & & & & & -0.06 & $(0.02)$ & 0.005 & -0.06 & $(0.03)$ & 0.012 \\
\hline
\end{tabular}

Note; in Model 1 individual favorable psychosocial factors were independently tested and in other models (2-3) simultaneously tested.

Combined favorable psychosocial factor is tested independently of individual psychosocial factors in both Models 4 and 5 . Bold values indicate significance at $\mathrm{p}<0.05$. 
promotion of other protective factors that help children and adolescents avoid multiple behaviors that place them at risk of adverse health and educational outcomes. These protective factors are individual or environmental characteristics, conditions, or behaviors (positive parenting, parental engagement, reducing inequality and socioeconomic instability threat) which reduce the effects of stressful life events. Our results offer further support for these recommendations.

The associations between childhood psychosocial factors and increased BMI were only marginally affected by age or sex. These associations were only slightly weakened after adjusting for adult socioeconomic status indicated by education. This finding suggests that socioeconomic status may be one of the mediators linking childhood psychosocial factors and adulthood BMI, thus shedding light on the mechanisms by which early life experiences influence health across the life course. Higher education may exert its influence through better health-related knowledge (e.g., about nutrition), better social or financial resources, and greater motivation to engage in healthier eating habits and physical activity patterns, thus leading to a slower increase in BMI. When using the imputed data sets, the association between positive childhood factors and a lower BMI was not totally explained by education, and thus this effect may be smaller than estimated when using the complete case data. This finding suggests that some selective attrition has occurred related to BMI. The other results were replicated when using the imputed data.

The immediate psychosocial environment exploits biological, psychological, social and economic vulnerability, which may also promote overconsumption of unhealthy foods. Our findings suggest that the accumulation of positive experiences may be associated with better possibilities of maintaining a lower body weight throughout the life course. Improved socioeconomic status may be one pathway connecting positive childhood factors with a lower BMI in adulthood, but future studies should examine other potential mediators between early-life factors and future obesity, as well as potential windows of opportunity for intervening at different stages of the life-course. Our results show that family-related psychosocial factors, and especially the accumulation of such factors, may have long-term effects on weight.

Our results also offer some support to the health-in-all-policies principle, suggesting that influential interventions focus on multiple factors at multiple levels. Our findings are also in line with policy statements that point to childhood as a critical period in setting up trajectories that will influence health and healthy aging over the life course (Garner et al., 2012). The existing evidence now suggests that factors influencing weight gain include genetic, individual, neighborhood, area and national-level factors and our results offer further evidence for the need for action in childhood and family-level settings. It seems reasonable to conclude that all childhood settings can contribute to the creation of healthy environments, improved diet and physical activity patterns, and healthy body size.

\subsection{Strengths and limitations}

The strengths of our multi-center study are its longitudinal study design from childhood to adulthood, the relatively large number of childhood factors measured prospectively in childhood (which eliminates the recall bias typically present in retrospective studies), and the precise measurement of weight by trained clinical personnel. The major limitation of our study is the considerable number of participants dropping out of the study; however, due to the multilevel design, we were also able to include participants with partially missing data, and analyses with the imputed data showed similar associations to those in the observed data. Our questionnaires on childhood psychosocial factors, while more comprehensive than most, were designed three decades ago for this specific study and are not widely used, which limits the possibility of comparing them with those of other studies. The cumulative psychosocial score was based on binary variables that are well-justified in some cases (e.g., diagnosis vs. no diagnosis) but have a less clear rationale in others. Cumulative binary scores have, however, the advantage of being parsimonious, they make no assumptions about the relative strengths of multiple risk factors or their collinearity, and they enable the testing of additive effects over a range of exposures (Evans et al., 2013). It has been shown that these psychosocial factor domains only moderately accumulate, but, when they do, such an accumulation of positive factors may be particularly strongly associated with later health outcomes (Slopen et al., 2013b). Finally, the sample was of European origin (Caucasian), thus representing a relatively homogenous population in a Western country. The results may not apply to ethnically or culturally different populations.

Because we were interested specifically in the accumulation of early life experiences, we did not include a wide range of potential later-life events or experiences in the analyses, and that is of course a limitation in our study. However, we did take into account education in adulthood as a measure of later socioeconomic status, which is a good marker of later psychosocial development, and this did not affect the associations significantly.

\section{Conclusions}

In this longitudinal analysis of a well-characterized, populationbased sample of apparently healthy adults, the health behaviors of parents and a lack of stressful life-events were associated with healthier BMI development over the lifespan, as well as a lower risk of obese BMI during adulthood. Positive early-life factors contribute to a healthy body weight, and this knowledge is essential for developing early life preventions and interventions aimed at reducing the obesity epidemic.

\section{Funding sources}

The Young Finns Study has been financially supported by the Academy of Finland: grants 286284 (T.L.), 134309 (Eye), 126925, 121584, 124282, 129378 (Salve), 117787 (Gendi), 41071 (Skidi) and 265977 (M.E.); the Social Insurance Institution of Finland; Kuopio, Tampere and Turku University Hospital Medical Funds (grant X51001 for T.L.); the Juho Vainio Foundation; the Paavo Nurmi Foundation; the Finnish Foundation for Cardiovascular Research; the Finnish Cultural Foundation; the Tampere Tuberculosis Foundation; the Emil Aaltonen Foundation; the Yrjö Jahnsson Foundation (T.L.); and the Signe and Ane Gyllenberg Foundation (to L.P.-R.)

\section{Financial disclosure}

The authors have no financial relationships relevant to this article to disclose.

\section{Conflict of interest}

The authors have no conflicts of interest to disclose.

\section{Contributors' statements}

Marko Elovainio conceptualized and designed the study, carried out the initial analyses, drafted the initial manuscript, and approved the final manuscript as submitted. Laura Pulkki-Råback and Markus Juonala conceptualized and designed the study, designed the data collection instruments, reviewed and revised the manuscript, and approved the final manuscript as submitted. Christian Hakulinen designed the analysis methods, reviewed and revised the manuscript, and approved the final manuscript as submitted. Olli Raitakari coordinated and supervised data collection, designed the data collection instruments, reviewed and revised the manuscript, and approved the final manuscript as submitted. Liisa Keltikangas-Järvinen designed the data collection instruments, coordinated and supervised data collection, reviewed and revised the manuscript, and approved the final manuscript as submitted. Terho 
Lehtimäki, Eero Jokinen, Tapani Rönnemaa, Vera Mikkilä, Päivi Tossavainen, Antti Jula, Nina Hutri-Kähönen, and Jorma Viikari reviewed and revised the manuscript, and approved the final manuscript as submitted.

\section{Transparency document}

The Transparency document associated with this article can be found, in the online version.

\section{Appendix A. Supplementary data}

Supplementary data to this article can be found online at http://dx. doi.org/10.1016/j.ypmed.2016.12.023.

\section{References}

Appleton, A.A., Buka, S.L., Loucks, E.B., Rimm, E.B., Martin, L.T., LD, K., 2013a. A prospective study of positive early-life psychosocial factors and favorable cardiovascular risk in adulthood. Circulation 127, 905-912.

Appleton, A.A., Buka, S.L., Loucks, E.B., Rimm, E.B., Martin, L.T., Kubzansky, L.D., 2013b. A prospective study of positive early-life psychosocial factors and favorable cardiovascular risk in adulthood. Circulation 127, 905-912.

Boehm, J.K., Kubzansky, L.D., 2012. The association between positive psychological wellbeing and cardiovascular health. Psychol. Bull. 138, 655-691.

Chida, Y., Steptoe, A., 2008. Positive psychological well-being and mortality: a quantitative review of prospective observational studies. Psychosom. Med. 70, 7741-7756.

Danese, A., Tan, M., 2014. Childhood maltreatment and obesity: systematic review and meta-analysis. Mol. Psychiatry 19, 544-554.

Evans, G.W., Dongping, L., Whipple, S.S., 2013. Cumulative risk and child development Psychol. Bull. 139, 1342-1396.

Flegal, K.M., Graubard, B.I., Williamson, D.F., Gail, M.H., 2005. Excess deaths associated with underweight, overweight, and obesity. JAMA 293, 1891-1896.

Garner, A.S., Shonkoff, J.P., Health CoPAoCaF, Committee on Early Childhood A, and Dependent Care; Section on Developmental and Behavioral Pediatrics, 2012. Early childhood adversity, toxic stress, and the role of the pediatrician: translating developmental science into lifelong health. Pediatrics 129, e224-e231.

Hägg, S., Fall, T., Ploner, A., et al., Consortium. ENfGaGE, 2015. Adiposity as a cause of cardiovascular disease: a Mendelian randomization study. Int. J. Epidemiol. 44 (2), 578-588.

Johnson, J.L., Leff, M., Johnson, J.L., Leff, M., 1999. Children of substance abusers: overview of research findings. Pediatrics 103, 1085-1099.
Katainen, S., Räikkönen, K., Keltikangas-Järvinen, L., 1997. Childhood temperament and mother's child-rearing attitudes: stability and interaction in a three-year follow-up study. Eur. J. Personal. 11, 249-265.

Keltikangas-Järvinen, L., Pulkki-Råback, L., Puttonen, S., Viikari, J., Raitakari, O.T., 2006. Childhood hyperactivity as a predictor of carotid artery intima media thickness over a period of 21 years: the Cardiovascular Risk in Young Finns Study. Psychosom. Med. 68, 509-516.

Korkeila, J Vahtera, J Korkeila, K, Kivimäki, M. Sumanen, M., Koskenvuo, K et al, 2010. Childhood adversities as predictors of incident coronary heart disease and cerebrovascular disease. Heart 96, 298-303.

Kubzansky, L.D., Park, N., Peterson, C., Vokonas, P., Sparrow, D., 2011. Healthy psychological functioning and incident coronary heart disease. Arch. Gen. Psychiatry 68, 400-408.

Mason, S.M., Austin, S.B., Bakalar, J.L., et al., 2016. Child maltreatment's heavy toll: the need for trauma-informed obesity prevention. Am. J. Prev. Med. 50 (5), 646-649.

Moffitt, T.E., Arseneault, L., Belsky, D., et al., 2011. A gradient of childhood self-control predicts health, wealth, and public safety. Proc. Natl. Acad. Sci. 108, 2693-2698.

Nishtar, S., Gluckman, P., Armstrong, T., 2014. Ending childhood obesity: a time for action. Lancet 387, 825-827.

Ogden, C.L., Flegal, K.M., Carroll, M.D., Johnson, C.L., 2002. Prevalence and trends in overweight among US children and adolescents, 1999-2000. J. Am. Med. Assoc. 288, 1728-1732.

Pulkki-Råback, L., Elovainio, M., Kivimäki, M., Raitakari, O.T., Keltikangas-Järvinen, L., 2005. Temperament in childhood predicts body mass in adulthood: the Cardiovascular Risk in Young Finns Study. Health Psychol. 24, 307-315.

Pulkki-Raback, L., Elovainio, M., Kivimaki, M., Raitakari, O.T., Keltikangas-Jarvinen, L., 2005. Temperament in childhood predicts body mass in adulthood: the Cardiovascular Risk in Young Finns Study. Health Psychol. 24 (3), 307-315.

Pulkki-Råback, L, Elovainio, M., Hakulinen, C., et al., 2015. Cumulative effect of psychosocial factors in youth on ideal cardiovascular health in adulthood. The Cardiovascular Risk in Young Finns Study. Circulation 131 (3):245-253. http://dx.doi.org/10.1161/ CIRCULATIONAHA.113.007104.

Raitakari, O.T., Juonala, M., Rönnemaa, T., et al., 2008. Cohort profile: the Cardiovascular Risk in Young Finns Study. Int. J. Epidemiol. 37, 1220-1226.

Slopen, N., Goodman, E., Koenen, K.C., Kubzansky, L.D., 2013a. Socioeconomic and other social stressors and biomarkers of cardiometabolic risk in youth: a systematic review of less studied risk factors. PLoS One 8, e64418.

Slopen, N., Goodman, E., Koenen, K.C., Kubzansky, L.D., 2013b. Socioeconomic and other social stressors and biomarkers of cardiometabolic risk in youth: a systematic review of less studied risk factors. PLoS One 8, e64418.

Vazquez, G., Duval, S., Jacobs, D.R., S, K., 2007. Comparison of body mass index, waist circumference, and waist/hip ratio in predicting incident diabetes: a meta-analysis. Epidemiol. Rev. 29, 115-128. 